\title{
Nonclassical interpolation in spaces of smooth functions
}

\author{
by \\ VLA DIMIR I. O V CHIN N IKOV (Voronezh)
}

\begin{abstract}
We prove that the fractional BMO space on a one-dimensional manifold is an interpolation space between $C$ and $C^{1}$. We also prove that $\mathrm{BMO}^{1}$ is an interpolation space between $C$ and $C^{2}$. The proof is based on some nonclassical interpolation constructions. The results obtained cannot be transferred to spaces of functions defined on manifolds of higher dimension. The interpolation description of fractional BMO spaces is used at the end of the paper for the proof of the boundedness of commutators of the Hilbert transform.
\end{abstract}

The aim of this paper is to prove the interpolation property of the fractional BMO space between $C$ and $C^{1}$, which was announced in [7]. It is well known that these fractional BMO spaces, being particular examples of Lizorkin-Triebel spaces, cannot be reduced even to the generalized Besov spaces. So we enlarge the family of interpolation spaces between $C$ and $C^{1}$.

The same can be applied to the pair $C$ and $C^{2}$ and we conclude that $\mathrm{BMO}^{1}$ is an interpolation space between $C$ and $C^{2}$. So we find a natural substitute for the space $C^{1}$, which itself turns out not to be an interpolation space between $C$ and $C^{2}$ (see [4]).

The dimension of the manifolds where our functions are defined is important. It turns out that the fractional BMO spaces on a 2-dimensional manifold are not interpolation spaces between the corresponding spaces of smooth functions generated by the $L_{\infty}$-metric.

The work consists of six sections. In the first section we describe the spaces whose interpolation properties are discussed in the sequel. In Section 2 we introduce the Hilbert interpolation functor. This interpolation construction is used in Section 3 in the proof of interpolation of the fractional BMO space between the spaces of bounded and Lipschitz functions. In Section 4 we consider other nonclassical interpolation constructions, which yield the same interpolation spaces. These results allow us to prove in Section 5 that the fractional BMO space is an interpolation space between the spaces of continuous and continuously differentiable functions. Finally, in

1991 Mathematics Subject Classification: 46E35, 46M35. 
Section 6 we consider one application of the functorial description of fractional BMO spaces to commutators of the Hilbert transform.

1. Spaces of smooth functions. Let $M$ be a one-dimensional Riemannian manifold with boundary, i.e. $M$ is either an interval, a semi-axis, the real axis, or a circle.

As usual we write $L_{\infty}(M)$ for the space of all bounded measurable functions on $M$ with the standard norm

$$
\|f\|_{L_{\infty}(M)}=\operatorname{ess~sup}_{t \in M}|f(t)| .
$$

If $k$ is a natural number, let $L_{\infty}^{k}(M)$ denote the space of functions whose generalized derivatives of order $k$ belong to $L_{\infty}(M)$. Denoting by $D$ the operator of differentiation, we see that $f \in L_{\infty}^{k}(M)$ if $D^{k} f \in L_{\infty}(M)$, and we introduce a norm on $L_{\infty}^{k}(M)$ by

$$
\|f\|_{L_{\infty}^{k}(M)}=\sum_{n=0}^{k-1}\left|f^{(n)}\left(t_{0}\right)\right|+\left\|D^{k} f\right\|_{L_{\infty}(M)}
$$

where $t_{0} \in M$.

The space $L_{\infty}^{k}(M)$ coincides with the space of functions such that $D^{k-1} f$ is a Lipschitz function, i.e.

$$
\sup _{s, t} \frac{\left|D^{k-1} f(t)-D^{k-1} f(s)\right|}{|t-s|}<\infty .
$$

Obviously, for an interval or a circle this space consists of bounded functions, so $L_{\infty}^{k}(M)$ coincides with the Sobolev space constructed with respect to the uniform metric. In the case of a noncompact manifold the space $L_{\infty}^{k}(M)$ contains unbounded functions, and $L_{\infty}(M) \cap L_{\infty}^{k}(M)=W_{\infty}^{k}(M)$ is not closed in $L_{\infty}^{k}(M)$.

If we use the standard duality defined by the integral

$$
\langle f, g\rangle=\int_{M} f(t) g(t) d t
$$

we see that $L_{\infty}(M)=L_{1}(M)^{*}$. Furthermore, Ciesielski and Figiel (see [3]) showed that $L_{\infty}^{k}(M)=\left(\stackrel{\circ}{W}_{1}^{-k}(M)\right)^{*}$, where $\stackrel{\circ}{W}_{1}^{-k}(M)$ is the closure of the set of smooth functions on $M$ which vanish in a neighborhood of the boundary and infinity, with respect to the norm

$$
\|g\|=\sup _{f} \int_{M} f(t) g(t) d t
$$

where the supremum is taken over all $f \in L_{\infty}^{k}(M)$ and $\|f\|_{L_{\infty}^{k}(M)} \leq 1$.

Hence $\left\{L_{\infty}(M), L_{\infty}^{k}(M)\right\}$ is dual to the pair $\left\{L_{1}(M), \stackrel{\circ}{W}_{1}^{-k}(M)\right\}$. 
Recall that the space $\mathrm{BMO}(\mathbb{R})$ consists of locally integrable functions $f$ on $\mathbb{R}$ such that

$$
\sup _{Q} \frac{1}{|Q|} \int_{Q}\left|f(t)-f_{Q}\right| d t<\infty \quad \text { where } \quad f_{Q}=\frac{1}{|Q|} \int_{Q} f(t) d t
$$

and $Q$ runs over all finite intervals in $\mathbb{R}$.

Let $\operatorname{bmo}(\mathbb{R})$ denote the nonhomogeneous BMO space (see [14]), which consists of functions such that

$$
\sup _{Q} \frac{1}{|Q|} \int_{Q}\left|f(t)-f_{Q}\right| d t<\infty \text { and } \sup _{|Q| \geq 1} \frac{1}{|Q|} \int_{Q}|f(t)| d t<\infty .
$$

Denote by $\operatorname{bmo}^{\alpha}(\mathbb{R})$ the space of Bessel potentials of order $\alpha$ generated by functions from $\operatorname{bmo}(\mathbb{R})$. That is, if we put, for $\alpha \in \mathbb{R}$,

$$
J_{\alpha} f=\mathcal{F}^{-1}\left(\left(1+|\xi|^{2}\right)^{-\alpha / 2} \widehat{f}(\xi)\right)
$$

where $\widehat{f}$ is the Fourier transform and $\mathcal{F}^{-1}$ is the inverse Fourier transform, then bmo $^{\alpha}(\mathbb{R})$ coincides with the image $J_{\alpha}(\mathrm{bmo})$.

It is shown in [12] that $f \in \mathrm{bmo}^{\alpha}(\mathbb{R})$ for $0<\alpha<1$ if and only if $f \in \operatorname{bmo}(\mathbb{R})$ and

$$
A_{1}=\sup _{Q} \frac{1}{|Q|} \int_{Q} \int_{|s| \leq|Q|} \frac{|f(t+s)-f(t)|^{2}}{|s|^{1+2 \alpha}} d s d t<\infty,
$$

and for $0<\alpha<2$ if and only if $f \in \operatorname{bmo}(\mathbb{R})$ and

$$
\text { (2) } \quad A_{2}=\sup _{Q} \frac{1}{|Q|} \int_{Q} \int_{|s| \leq|Q|} \frac{|f(t+2 s)-2 f(t+s)+f(t)|^{2}}{|s|^{1+2 \alpha}} d s d t<\infty
$$

where $Q$ runs over all finite intervals in $\mathbb{R}$.

As the conditions (1) and (2) have metric character, they can be formulated on a Riemannian manifold $M$. This allows us to consider the space bmo $^{\alpha}(M)$ of functions $f \in \operatorname{bmo}(M)$ satisfying (1) or (2) on the corresponding manifold, equipped with the norm

$$
\|f\|_{\mathrm{bmo}^{\alpha}(M)}=\|f\|_{\mathrm{bmo}(M)}+A_{1}
$$

for $0<\alpha<1$, or

$$
\|f\|_{\mathrm{bmo}^{\alpha}(M)}=\|f\|_{\mathrm{bmo}(M)}+A_{2}
$$

for $0<\alpha<2$.

It is well known (see [14]) that $\mathrm{bmo}^{\alpha}(\mathbb{R}) \subset L_{\infty}(\mathbb{R})$ for $\alpha>0$. Thus, conditions (1) or (2) plus boundedness mean that the corresponding functions belong to $\mathrm{bmo}^{\alpha}(\mathbb{R})$. A similar statement is true for $\mathrm{bmo}^{\alpha}(M)$. Note also that $\mathrm{bmo}^{k \theta}(M)$ turns out to be an intermediate space between $L_{\infty}(M)$ and $L_{\infty}^{k}(M)$, and between $L_{\infty}(M)$ and $L_{\infty}^{k}(M) \cap L_{\infty}(M)$ if $0<\theta<1$, that is,

$$
L_{\infty}^{k}(M) \cap L_{\infty}(M) \subset \operatorname{bmo}^{k \theta}(M) \subset L_{\infty}(M) .
$$


2. The Hilbert functor. In the sequel we use standard notions and notation from interpolation theory (see, for instance, [1]). So let $\bar{X}=\left\{X_{0}, X_{1}\right\}$ denote any Banach pair.

Let $\bar{H}=\left\{H_{0}, H_{1}\right\}$ be any pair of Hilbert spaces. We denote by $H_{\theta}$ an intermediate space of this pair constructed by the Calderón complex method of interpolation, that is, $H_{\theta}=\left[H_{0}, H_{1}\right]_{\theta}$. It is known that the space $H_{\theta}$ turns out to be a Hilbert space, and the family $H_{\theta}$, where $0 \leq \theta \leq 1$, is a unique scale of Hilbert spaces connecting $H_{0}$ and $H_{1}$. The same scale can also be obtained by the Lions-Peetre construction, i.e. $H_{\theta}=\left(H_{0}, H_{1}\right)_{\theta, 2}$ with equivalent norms.

DeFinition. By the upper Hilbert interpolation functor we mean the maximal extension of the functor $H_{\theta}=\mathcal{F}_{\theta}\left(H_{0}, H_{1}\right)$ from the category of all Hilbert pairs to the category of all Banach pairs. We denote it by $\left\langle X_{0}, X_{1}\right\rangle^{\theta, 2}$.

In other words, $x$ belongs to $\left\langle X_{0}, X_{1}\right\rangle^{\theta, 2}$ if $x \in X_{0}+X_{1}$ and

$$
\sup _{T}\|T x\|_{H_{\theta}}<\infty
$$

where the supremum is taken over all linear operators mapping $\bar{X}$ into any Hilbert pair $\bar{H}$ and $\|T\|_{X_{i} \rightarrow H_{i}} \leq 1(i=0,1)$.

The maximality and the equalities $H_{\theta}=\left[H_{0}, H_{1}\right]^{\theta}=\left(H_{0}, H_{1}\right)_{\theta, 2}$ imply at once the imbeddings of $\left(X_{0}, X_{1}\right)_{\theta, 2}$ and $\left[X_{0}, X_{1}\right]^{\theta}$ into $\left\langle X_{0}, X_{1}\right\rangle^{\theta, 2}$. The upper Hilbert functor yields a power transformation of weights for onedimensional pairs. This means that the characteristic function of this functor is equal to $s^{1-\theta} t^{\theta}$. Hence $\left\langle X_{0}, X_{1}\right\rangle^{\theta, 2} \subset\left(X_{0}, X_{1}\right)_{\theta, \infty}$. It is easy to see that no imbedding mentioned above can be improved on the category of all Banach pairs.

The description of the spaces $\left\langle X_{0}, X_{1}\right\rangle^{\theta, 2}$ by means of all pairs of Hilbert spaces may happen to be inconvenient. Obviously, the following reductions to finite-dimensional weight pairs are possible.

Denote by $l_{2}^{N}\left(2^{-n \theta}\right)$ where $0 \leq \theta \leq 1$ and $N \in \mathbb{N}$ the space of sequences $\left\{\xi_{n}\right\}_{n=-N}^{N}$ with the norm

$$
\left(\sum_{n=-N}^{N}\left(\left|\xi_{n}\right| 2^{-\theta n}\right)^{2}\right)^{1 / 2}
$$

Proposition 1. The space $\left\langle X_{0}, X_{1}\right\rangle^{\theta, 2}$ consists of those $x \in X_{0}+X_{1}$ for which

$$
\sup \|U(x)\|_{l_{2}^{N}\left(2^{-n \theta}\right)}<\infty
$$

where the supremum is taken over all $N \in \mathbb{N}$ and linear operators $U: \bar{X} \rightarrow$ $\left\{l_{2}^{N}, l_{2}^{N}\left(2^{-n}\right)\right\}$ such that $\|U\|_{X_{i} \rightarrow l_{2}^{N}\left(2^{-n i}\right)} \leq 1$ for $i=0,1$. 
Proposition 2. Assume that a pair $\left\{X_{0}, X_{1}\right\}$ is dual, i.e. $X_{i}=Y_{i}^{*}$ $(i=0,1)$ where $\left\{Y_{0}, Y_{1}\right\}$ is a regular Banach pair. Then $x \in\left\langle X_{0}, X_{1}\right\rangle^{\theta, 2}$ if

$$
\sup _{N, f_{n}} \sum_{n=-N}^{N}\left(2^{-n \theta}\left|f_{n}(x)\right|\right)^{2}<\infty
$$

where $f_{n} \in Y_{0} \cap Y_{1}$ is such that

$$
\sup _{\|z\|_{X_{i}} \leq 1} \sum_{n=-N}^{N}\left(2^{-n i}\left|f_{n}(z)\right|\right)^{2} \leq 1
$$

for $i=0,1$.

In other words, it is possible to take the supremum in Proposition 1 over dual operators only.

Finally, we consider one more restriction on the set of operators which are used in the construction of the Hilbert functor.

Proposition 3. If a pair $\left\{X_{0}, X_{1}\right\}$ is dual, i.e. $X_{i}=Y_{i}^{*}(i=0,1)$ where $\left\{Y_{0}, Y_{1}\right\}$ is a regular Banach pair, and $K \subset X_{0} \cap X_{1}$ is a finite-dimensional subspace, then $x \in\left\langle X_{0}, X_{1}\right\rangle^{\theta, 2}$ if

$$
\sup _{N, f_{n}} \sum_{n=-N}^{N}\left(2^{-n \theta}\left|f_{n}(x)\right|\right)^{2}<\infty,
$$

where $f_{n} \in Y_{0} \cap Y_{1}, f_{n}(K)=0$, and

$$
\sup _{\|z\|_{X_{i}} \leq 1} \sum_{n=-N}^{N}\left(2^{-n i}\left|f_{n}(z)\right|\right)^{2} \leq 1
$$

for $i=0,1$.

3. Interpolation theorem. We now apply the upper Hilbert interpolation functor to interpolation of fractional bmo spaces.

Theorem 1. For any one-dimensional Riemannian manifold $M$ and any $0<\theta<1, k=1,2$ we have

$$
\left\langle L_{\infty}(M), L_{\infty}^{k}(M)\right\rangle^{\theta, 2} \cap L_{\infty}(M)=\mathrm{bmo}^{k \theta}(M) .
$$

Proof. We give the proof only for the case $k=1$; the case $k=2$ is left to the reader. Let $Q$ be any interval in $M$. Let us introduce a Hilbert pair $\left\{H_{0}, H_{1}\right\}$, corresponding to $Q$, where $H_{0}=L_{2}(Q) / \mathbb{R}$ is the quotient space with respect to the subspace of constants, and the norm in $H_{0}$ is generated by the integral

$$
\left(\frac{1}{|Q|} \int_{Q}|x(t)|^{2} d t\right)^{1 / 2}
$$


The space $H_{1}$ is $W_{2}^{1}(Q) / \mathbb{R}$ with the norm generated by the integral

$$
\left(\frac{1}{|Q|} \int_{Q}\left|x^{\prime}(t)\right|^{2} d t\right)^{1 / 2} \text {. }
$$

It is obvious that the pair $\left\{L_{\infty}(M), L_{\infty}^{1}(M)\right\}$ is mapped into $\left\{H_{0}, H_{1}\right\}$. If we write $\widetilde{x}$ for the element of a quotient space corresponding to $x$, then

$$
\begin{aligned}
\|\widetilde{x}\|_{H_{0}} & \leq\|x\|_{L_{2}(Q)} \leq\|x\|_{L_{\infty}(M)}, \\
\|\widetilde{x}\|_{H_{1}} & \leq \sup _{t}\left|x^{\prime}(t)\right| \leq\|x\|_{L_{\infty}^{1}(M)} .
\end{aligned}
$$

Hence, by definition of the Hilbert functor,

$$
\sup _{Q}\|\widetilde{x}\|_{\left(H_{0}, H_{1}\right)_{\theta, 2}} \leq c\|x\|_{\left\langle L_{\infty}(M), L_{\infty}^{k}(M)\right\rangle^{\theta, 2} .}
$$

For a unit interval $Q$, the norm in the space $\left(H_{0}, H_{1}\right)_{\theta, 2}$ can be expressed in terms of differences, i.e.

$$
\|\widetilde{x}\|_{\left(H_{0}, H_{1}\right)_{\theta, 2}}^{2} \asymp \iint_{Q Q} \frac{|x(t)-x(s)|^{2}}{|t-s|^{1+2 \theta}} d s d t .
$$

In view of the homogeneity of the expressions (3)-(5) with respect to dilation we find that for any interval $Q$,

$$
\|\widetilde{x}\|_{\left(H_{0}, H_{1}\right)_{\theta, 2}}^{2} \asymp \frac{1}{|Q|} \iint_{Q Q} \frac{|x(t)-x(s)|^{2}}{|t-s|^{1+2 \theta}} d s d t,
$$

where the constants of equivalence are independent of the intervals $Q$. Thus,

$$
\sup _{Q} \frac{1}{|Q|} \iint_{Q Q} \frac{|x(t)-x(s)|^{2}}{|t-s|^{1+2 \theta}} d s d t \leq c\|x\|_{\left\langle L_{\infty}(M), L_{\infty}^{k}(M)\right\rangle^{\theta, 2}}^{2} .
$$

Hence, by (1),

$$
\left\langle L_{\infty}(M), L_{\infty}^{1}(M)\right\rangle^{\theta, 2} \cap L_{\infty}(M) \subset \operatorname{bmo}^{\theta}(M)
$$

for $0<\theta<1$. Similarly using $(2)$ we find

$$
\left\langle L_{\infty}(M), L_{\infty}^{2}(M)\right\rangle^{\theta, 2} \cap L_{\infty}(M) \subset \operatorname{bmo}^{2 \theta}(M) .
$$

It remains to prove the converse imbedding

$$
\mathrm{bmo}^{k \theta}(M) \subset\left\langle L_{\infty}(M), L_{\infty}^{k}(M)\right\rangle^{\theta, 2} .
$$

Again we consider only the case $k=1$, and we show that if

$$
\sup _{Q} \frac{1}{|Q|} \iint_{Q Q} \frac{|x(t)-x(s)|^{2}}{|t-s|^{1+2 \theta}} d s d t<\infty
$$

and $x \in L_{\infty}(M)$, then

$$
\sup _{T}\|T(x)\|_{H_{\theta}}<\infty
$$


where the supremum is taken over all bounded linear operators

$$
T:\left\{L_{\infty}(M), L_{\infty}^{1}(M)\right\} \rightarrow\left\{H_{0}, H_{1}\right\}
$$

with unit norms. In view of Proposition 3 we can consider only operators which are dual and equal to zero at constants. Let $T$ be such an operator.

Dual bounded operators mapping $L_{\infty}(M)$ into a Hilbert space have a remarkable factorization property (see, for example, [9]): for any $T$ : $L_{\infty}(M) \rightarrow H_{0}$ there exists $g_{0} \in L_{1}(M)$ such that

$$
\|T x\|_{H_{0}}^{2} \leq \int_{M}|x(t)|^{2} g_{0}(t) d t
$$

where $\left\|g_{0}\right\|_{L_{1}(M)} \leq c\|T\|_{L_{\infty}(M) \rightarrow H_{0}}$ ( $c$ is a universal constant). Obviously, a similar statement is also true for operators mapping from $L_{\infty}^{1}(M)$ into a Hilbert space. For any dual bounded linear operator $T: L_{\infty}^{1}(M) \rightarrow H_{1}$ with $\operatorname{Ker} T \supset \operatorname{Ker} D$ there exists $g_{1} \in L_{1}(M)$ such that

$$
\|T x\|_{H_{1}}^{2} \leq \int_{M}|D x(t)|^{2} g_{1}(t) d t
$$

where $\left\|g_{1}\right\|_{L_{1}(M)} \leq c\|T\|_{L_{\infty}^{1}(M) \rightarrow H_{1}}$.

If we put $g=\max \left\{\left|g_{0}\right|,\left|g_{1}\right|\right\}$, then

$$
\|T x\|_{H_{0}} \leq c_{0}\|x\|_{L_{2}(g)} \quad \text { and } \quad\|T x\|_{H_{1}} \leq c_{1}\|D x\|_{L_{2}(g)} .
$$

Let $\widetilde{L}_{2}(g)$ denote the quotient space $L_{2}(g) / \mathbb{R}$ where $\mathbb{R}$ is the subspace of constant functions. If $W_{2}^{1}(g)$ denotes the space of functions $x$ such that $D x \in L_{2}(g)$, let $\widetilde{W}_{2}^{1}(g)$ denote the quotient space $W_{2}^{1}(g) / \mathbb{R}$, equipped with the norm $\|\widetilde{x}\|_{\widetilde{W}_{2}^{1}(g)}=\|D x\|_{L_{2}(g)}$. (Here again $\widetilde{x}$ denotes the element of the quotient space corresponding to $x$.) Thus,

$$
\|T x\|_{H_{0}}=\|T \widetilde{x}\|_{H_{0}} \leq c_{0}\|\widetilde{x}\|_{\widetilde{L}_{2}(g)}, \quad\|T x\|_{H_{1}}=\|T \widetilde{x}\|_{H_{1}} \leq c_{1}\|\widetilde{x}\|_{\widetilde{W}_{2}^{1}(g)} .
$$

Interpolating by the Lions-Peetre method, we see that

$$
\|T x\|_{\left(H_{0}, H_{1}\right)_{\theta, 2}} \leq c_{0}^{1-\theta} c_{1}^{\theta}\|\widetilde{x}\|_{\left(\widetilde{L}_{2}(g), \widetilde{W}_{2}^{1}(g)\right)_{\theta, 2}} .
$$

Thus

$$
\sup _{\|T\| \leq 1}\|T x\|_{\left(H_{0}, H_{1}\right)_{\theta, 2}} \leq c \sup _{\|g\|_{L_{1}} \leq 1}\|\widetilde{x}\|_{\left(\widetilde{L}_{2}(g), \widetilde{W}_{2}^{1}(g)\right)_{\theta, 2}} .
$$

It is well known that any integrable nonnegative function may be majorized by a function of class $C^{+}$, i.e. by a function which is the limit of an increasing sequence of simple functions. Each function from $C^{+}$in turn can be majorized by a function which is the limit of an increasing strictly positive sequence of quasi-simple functions of the form

$$
h(t)=\sum_{m=1}^{\infty} c_{m} \chi_{Q_{m}}(t)
$$


where $Q_{m}$ are disjoint intervals and the length of any $Q_{m}$ except a finite number is 1 . Denote by $C^{++}$this new class of functions.

Thus we see that

$$
\sup _{\|T\| \leq 1}\|T x\|_{\left(H_{0}, H_{1}\right)_{\theta, 2}} \leq c \sup _{\substack{h \in C^{++} \\\|h\|_{L_{1}} \leq 1}}\|\widetilde{x}\|_{\left(\widetilde{L}_{2}(h), \widetilde{W}_{2}^{1}(h)\right)_{\theta, 2}} .
$$

We claim that

$$
\sup _{\|T\| \leq 1}\|T x\|_{\left(H_{0}, H_{1}\right)_{\theta, 2}} \leq c \sup _{\|h\|_{L_{1}} \leq 1}\|\widetilde{x}\|_{\left(\widetilde{L}_{2}(h), \widetilde{W}_{2}^{1}(h)\right)_{\theta, 2}},
$$

where $h$ are quasi-simple functions. Indeed, let $h_{n}$ denote a sequence of quasi-simple functions which monotonically converges to $h \in C^{++}$and $\|h\|_{L_{1}} \leq 1$. It is obvious that $\|x\|_{L_{2}\left(h_{n}\right)} \rightarrow\|x\|_{L_{2}(h)}$, and consequently $\|\widetilde{x}\|_{\widetilde{L}_{2}\left(h_{n}\right)} \rightarrow\|\widetilde{x}\|_{\widetilde{L}_{2}(h)}$, as well as $\left\|x^{\prime}\right\|_{L_{2}\left(h_{n}\right)} \rightarrow\left\|x^{\prime}\right\|_{L_{2}(h)}$. We now show the equality

$$
\|\widetilde{x}\|_{\left(\widetilde{L}_{2}(h), \widetilde{W}_{2}^{1}(h)\right)_{\theta, 2}}=\lim _{n \rightarrow \infty}\|\widetilde{x}\|_{\left(\widetilde{L}_{2}\left(h_{n}\right), \widetilde{W}_{2}^{1}\left(h_{n}\right)\right)_{\theta, 2}} .
$$

All the spaces in this formula are continuously imbedded into $\widetilde{L}_{2}\left(h_{1}\right)+$ $\widetilde{W}_{2}^{1}\left(h_{1}\right)$. Denote by $S^{0}$ the unit ball in $\widetilde{L}_{2}(h)$, and by $S_{n}^{0}$ the unit ball in $\widetilde{L}_{2}\left(h_{n}\right)$. Correspondingly, let $S^{1}$ denote the unit ball in $\widetilde{W}_{2}^{1}(h)$, and $S_{n}^{1}$ the unit ball in $\widetilde{W}_{2}^{1}\left(h_{n}\right)$. All these unit balls are weakly closed in the unit ball of $\widetilde{L}_{2}\left(h_{1}\right)+\widetilde{W}_{2}^{1}\left(h_{1}\right)$, therefore all of them are metric compacta.

By assumption,

$$
\bigcap_{n=1}^{\infty} S_{n}^{0}=S^{0} \quad \text { and } \quad \bigcap_{n=1}^{\infty} \frac{1}{t} S_{n}^{1}=\frac{1}{t} S^{1}
$$

for all $t>0$. Therefore

$$
\operatorname{co}\left(S^{0} \cup \frac{1}{t} S^{1}\right)=\bigcap_{n=1}^{\infty} \operatorname{co}\left(S_{n}^{0} \cup \frac{1}{t} S_{n}^{1}\right)
$$

where $\operatorname{co}(A)$ is the convex hull of $A$.

Since $\operatorname{co}\left(S_{n}^{0} \cup t^{-1} S_{n}^{1}\right)$ and $\operatorname{co}\left(S^{0} \cup t^{-1} S^{1}\right)$ are the unit balls of the $K$ functionals

$$
K\left(t, \widetilde{x} ;\left\{\widetilde{L}_{2}\left(h_{n}\right), \widetilde{W}_{2}^{1}\left(h_{n}\right)\right\}\right) \quad \text { and } \quad K\left(t, \widetilde{x} ;\left\{\widetilde{L}_{2}(h), \widetilde{W}_{2}^{1}(h)\right\}\right),
$$

(7) implies

$$
K\left(t, \widetilde{x} ;\left\{\widetilde{L}_{2}\left(h_{n}\right), \widetilde{W}_{2}^{1}\left(h_{n}\right)\right\}\right) \rightarrow K\left(t, \widetilde{x},\left\{\widetilde{L}_{2}(h), \widetilde{W}_{2}^{1}(h)\right\}\right)
$$

for $\widetilde{x} \in \widetilde{L}_{2}(h)+\widetilde{W}_{2}^{1}(h)$, and the convergence is monotone. Therefore 


$$
\begin{aligned}
\int_{0}^{\infty}\left(\frac{K\left(t, \widetilde{x} ;\left\{\widetilde{L}_{2}\left(h_{n}\right), \widetilde{W}_{2}^{1}\left(h_{n}\right)\right\}\right)}{t^{\theta}}\right)^{2} & \frac{d t}{t} \\
& \rightarrow \int_{0}^{\infty}\left(\frac{K\left(t, \widetilde{x},\left\{\widetilde{L}_{2}(h), \widetilde{W}_{2}^{1}(h)\right\}\right)}{t^{\theta}}\right)^{2} \frac{d t}{t} .
\end{aligned}
$$

Thus

Hence in view of $(6)$,

$$
\|\widetilde{x}\|_{\left(\widetilde{L}_{2}\left(h_{n}\right), \widetilde{W}_{2}^{1}\left(h_{n}\right)\right)_{\theta, 2}} \rightarrow\|\widetilde{x}\|_{\left(\widetilde{L}_{2}(h), \widetilde{W}_{2}^{1}(h)\right)_{\theta, 2}} .
$$

$$
\sup _{\|T\| \leq 1}\|T x\|_{\left(H_{0}, H_{1}\right)_{\theta, 2}} \leq c \sup _{\|h\|_{L_{1}} \leq 1}\|\widetilde{x}\|_{\left(\widetilde{L}_{2}(h), \widetilde{W}_{2}^{1}(h)\right)_{\theta, 2}}
$$

where $h$ are quasi-simple functions.

Since

$$
h(t)=\sum_{m=1}^{\infty} c_{m} \chi_{Q_{m}}(t)=\sum_{m=1}^{\infty} c_{m}\left|Q_{m}\right| \frac{\chi_{Q_{m}}(t)}{\left|Q_{m}\right|}
$$

where $Q_{m}$ are disjoint intervals and $\sum_{m=1}^{\infty} c_{m}\left|Q_{m}\right| \leq 1$, we find

$$
\begin{aligned}
\|x\|_{L_{2}(h)}^{2} & =\int_{M}|x(t)|^{2} h(t) d t=\sum_{m=1}^{\infty} c_{m}\left|Q_{m}\right| \frac{1}{\left|Q_{m}\right|} \int_{Q_{m}}|x(t)|^{2} d t \\
& \leq \sup _{Q^{q}} \frac{1}{q} \int_{Q^{q}}|x(t)|^{2} d t
\end{aligned}
$$

where the supremum is taken over all intervals $Q^{q}$ of a fixed length $q \leq$ $\min _{m}\left|Q_{m}\right|$.

Similarly,

$$
\left\|x^{\prime}\right\|_{L_{2}(h)}^{2} \leq \sup _{Q^{q}} \frac{1}{q}\left\|x^{\prime}\right\|_{L_{2}\left(Q^{q}\right)}^{2}
$$

where the supremum is taken over the same intervals.

Denote by $\|x \mid\|_{W_{2}^{\alpha}(Q)}$ a norm on the Sobolev space $W_{2}^{\alpha}(Q)$ which is homogeneous with respect to dilation. For instance,

$$
\left\|\left.|| x\left|\|_{W_{2}^{\alpha}(Q)}^{2}=\right| Q\right|^{2 \alpha-1} \int_{Q}|x(t)|^{2} d t+\frac{1}{|Q|} \iint_{Q Q} \frac{|x(t)-x(s)|^{2}}{|t-s|^{1+2 \alpha}} d t d s\right.
$$

for $0<\alpha<1$, and similarly for other $\alpha \notin \mathbb{N}$; and

$$
\left.\|\| x\left|\|_{W_{2}^{\alpha}(Q)}^{2}=\right| Q\right|^{2 \alpha-1} \int_{Q}|x(t)|^{2} d t+\frac{1}{|Q|} \int_{Q}\left|x^{(\alpha)}(t)\right|^{2} d t
$$

for $\alpha \in \mathbb{N}$.

Let $S W_{2}^{\alpha}(M, q)$ denote the space of functions for which the norm

$$
\|x\|_{S W_{2}^{\alpha}(M, q)}=\sup _{Q^{q}}\|x\|_{W_{2}^{\alpha}\left(Q^{q}\right)}
$$


is finite. So (8) implies

$$
\|\widetilde{x}\|_{\widetilde{L}_{2}(h)} \leq\|x\|_{S W_{2}^{0}(M, q)},
$$

and (9) implies

$$
\begin{aligned}
\|\widetilde{x}\|_{\widetilde{W}_{2}^{1}(h)}^{2} & =\left\|x^{\prime}\right\|_{L_{2}(h)}^{2} \leq c \sup _{Q^{q}} \frac{1}{q}\left(q^{2}\|x\|_{L_{2}\left(Q^{q}\right)}^{2}+\left\|x^{\prime}\right\|_{L_{2}\left(Q^{q}\right)}^{2}\right) \\
& =c\|x\|_{S W_{2}^{1}(M, q)}^{2} .
\end{aligned}
$$

Interpolating these estimates, we conclude that

$$
\|\widetilde{x}\|_{\mathcal{F}\left(\widetilde{L}_{2}(h), \widetilde{W}_{2}^{1}(h)\right)} \leq c\|x\|_{\mathcal{F}\left(S W_{2}^{0}(M, q), S W_{2}^{1}(M, q)\right)}
$$

for any exact interpolation functor $\mathcal{F}$.

We shall use the interpolation relations between spaces $S W_{2}^{\alpha}(M, q)$, which can be described with the help of the second complex interpolation method of Calderón. Namely, we have

Lemma 1. For any $0<\theta<1$,

$$
\left[S W_{2}^{0}(M, q), S W_{2}^{k}(M, q)\right]^{\theta}=S W_{2}^{\theta k}(M, q),
$$

where the equivalence of the corresponding norms is uniform with respect to $q$ and $M$.

The proof is rather standard, so we omit it. However it is worth pointing out that homogeneity of the norms considered is essential.

Thus for $\mathcal{F}\left(X_{0}, X_{1}\right)=\left[X_{0}, X_{1}\right]^{\theta}$ in (10) we have

$$
\|\widetilde{x}\|_{\left[\widetilde{L}_{2}(h), \widetilde{W}_{2}^{1}(h)\right]^{\theta}} \leq c\|x\|_{S W_{2}^{\theta}(M, q)} .
$$

Since for any Hilbert pair, the spaces $\left(H_{0}, H_{1}\right)_{\theta, 2}$ and $\left[H_{0}, H_{1}\right]^{\theta}$ coincide, and the constants of equivalence of norms depend only on $\theta$, we see that

$$
\begin{aligned}
\sup _{h}\|\widetilde{x}\|_{\left(\widetilde{L}_{2}\left(h_{n}\right), \widetilde{W}_{2}^{1}\left(h_{n}\right)\right)_{\theta, 2}}^{2} & \leq c \sup _{|Q| \leq 1}|Q|^{2 \theta-1} \int_{Q}|x(t)|^{2} d t+\frac{1}{|Q|} \iint_{Q Q} \frac{|x(t)-x(s)|^{2}}{|t-s|^{1+2 \theta}} d s d t \\
& \leq c \sup _{Q} \frac{1}{|Q|} \int_{Q}|x(t)|^{2} d t+\sup _{Q} \frac{1}{|Q|} \iint_{Q Q} \frac{|x(t)-x(s)|^{2}}{|t-s|^{1+2 \theta}} d s d t .
\end{aligned}
$$

If $x \in \mathrm{bmo}^{\theta}(M)$, then both terms on the right hand side are obviously finite. Hence

$$
\operatorname{bmo}^{\theta}(M) \subset\left\langle L_{\infty}(M), L_{\infty}^{1}(M)\right\rangle^{\theta, 2},
$$

and

$$
\left\langle L_{\infty}(M), L_{\infty}^{1}(M)\right\rangle^{\theta, 2} \cap L_{\infty}(M)=\operatorname{bmo}^{\theta}(M) .
$$


Similarly

$$
\left\langle L_{\infty}(M), L_{\infty}^{2}(M)\right\rangle^{\theta, 2} \cap L_{\infty}(M)=\mathrm{bmo}^{2 \theta}(M) .
$$

The theorem is proved.

4. Other interpolation constructions. The upper Hilbert interpolation functor was used in the proof of the interpolation property of the fractional BMO space because it looks natural in the given situation. However, this functor is not well studied, so it would be natural to consider alternative functors which yield the same interpolation spaces.

The Lions-Peetre construction, applied to the pair $\left\{L_{\infty}(M), L_{\infty}^{k}(M)\right\}$, yields the Besov spaces. The fractional BMO spaces are not among them. The structure of the complex method spaces $\left[L_{\infty}(M), L_{\infty}^{k}(M)\right]_{\theta}$ remains an open problem. So we analyze the interpolation functors connected with the method of orbits.

We consider the interpolation constructions $\varphi_{m}\left(X_{0}, X_{1}\right), \varphi_{u}\left(X_{0}, X_{1}\right)$, and $G_{5}^{\varphi}\left(X_{0}, X_{1}\right)$, corresponding to function parameters $\varphi$ (see, for example, [5] or [6]).

Recall the definition of the spaces $\varphi_{u}\left(X_{0}, X_{1}\right)$ in the case of $\varphi(s, t)=$ $s^{1-\theta} t^{\theta}$ where $0<\theta<1$.

It is well known that $l_{1}\left(w_{0}^{1-\theta} w_{1}^{\theta}\right)$ is an interpolation space between $l_{1}\left(w_{0}\right)$ and $l_{1}\left(w_{1}\right)$, and $\left\{l_{1}\left(w_{0}\right), l_{1}\left(w_{1}\right)\right\} \mapsto l_{1}\left(w_{0}^{1-\theta} w_{1}^{\theta}\right)$ is an interpolation functor on the category of all pairs of the form $\left\{l_{1}\left(w_{0}\right), l_{1}\left(w_{1}\right)\right\}$ with arbitrary weights. The maximal extension of this functor to the category of all Banach pairs is denoted by $\varphi_{u}\left(X_{0}, X_{1}\right)$. Recall that $x \in \varphi_{u}\left(X_{0}, X_{1}\right)$ if

$$
\sup _{w_{0}, w_{1}, T}\|T x\|_{l_{1}\left(w_{0}^{1-\theta} w_{1}^{\theta}\right)}<\infty
$$

where the supremum is taken over all weights $w_{0}, w_{1}$ and all operators $T$ : $\left\{X_{0}, X_{1}\right\} \rightarrow\left\{l_{1}\left(w_{0}\right), l_{1}\left(w_{1}\right)\right\}$ such that $\|T\|_{X_{i} \rightarrow l_{1}\left(w_{i}\right)} \leq 1$ for $i=0,1$.

For any Hilbert pair $\left\{H_{0}, H_{1}\right\}$ we have $\varphi_{u}\left(H_{0}, H_{1}\right)=H_{\theta}$ (see [6]), therefore

where $\varphi(s, t)=s^{1-\theta} t^{\theta}$.

$$
\varphi_{u}\left(X_{0}, X_{1}\right) \subset\left\langle X_{0}, X_{1}\right\rangle^{\theta, 2}
$$

Proposition 4. For any $0<\theta<1$ we have

$$
\varphi_{u}\left(L_{\infty}(M), L_{\infty}^{k}(M)\right)=\left\langle L_{\infty}(M), L_{\infty}^{k}(M)\right\rangle^{\theta, 2}
$$

where $\varphi(s, t)=s^{1-\theta} t^{\theta}$.

Proof. Let $T:\left\{L_{\infty}(M), L_{\infty}^{k}(M)\right\} \rightarrow\left\{l_{1}\left(w_{0}\right), l_{1}\left(w_{1}\right)\right\}$ with unit norm. By Grothendieck's theorem the operators $T: L_{\infty}(M) \rightarrow l_{1}\left(w_{0}\right)$ and $T:$ $L_{\infty}^{k}(M) \rightarrow l_{1}\left(w_{1}\right)$ can be factorized through Hilbert spaces. This implies (see [6]) that $T$ can be factorized through a Hilbert pair $\left\{H_{0}, H_{1}\right\}$, that is, 
$T=S_{2} S_{1}$ where $S_{1}:\left\{L_{\infty}(M), L_{\infty}^{k}(M)\right\} \rightarrow\left\{H_{0}, H_{1}\right\}$ and $S_{2}:\left\{H_{0}, H_{1}\right\} \rightarrow$ $\left\{l_{1}\left(w_{0}\right), l_{1}\left(w_{1}\right)\right\}$.

If now $x \in\left\langle L_{\infty}(M), L_{\infty}^{k}(M)\right\rangle^{\theta, 2}$, then $S_{1}(x) \in H_{\theta}$. Hence $T(x)=$ $S_{2}\left(S_{1}(x)\right) \in l_{1}\left(w_{0}^{1-\theta} w_{1}^{\theta}\right)$, and

$$
\|T(x)\|_{l_{1}\left(w_{0}^{1-\theta} w_{1}^{\theta}\right)} \leq C\|x\|_{\left\langle L_{\infty}(M), L_{\infty}^{k}(M)\right\rangle^{\theta, 2} .}
$$

Thus $x \in \varphi_{u}\left(L_{\infty}(M), L_{\infty}^{k}(M)\right)$. Therefore

$$
\left\langle L_{\infty}(M), L_{\infty}^{k}(M)\right\rangle^{\theta, 2} \subset \varphi_{u}\left(L_{\infty}(M), L_{\infty}^{k}(M)\right) .
$$

The proposition is proved.

Now we turn to the functor $G_{5}^{\varphi}\left(X_{0}, X_{1}\right)$ (see [6]) where $\varphi(s, t)=s^{1-\theta} t^{\theta}$. The space $G_{5}^{\varphi}\left(X_{0}, X_{1}\right)$ can be described, for example, in terms of orbits as follows: $x \in G_{5}^{\varphi}\left(X_{0}, X_{1}\right)$ if $x=T\left(a_{\theta}\right)$ where $a_{\theta}=\left\{2^{n \theta}\right\}_{n=-\infty}^{\infty}$ and $T$ : $\left\{l_{1}, l_{1}\left(2^{-n}\right)\right\} \rightarrow\left\{X_{0}, X_{1}\right\}$ is such that $T^{*}: X_{0}^{*} \rightarrow l_{\infty}$ and $T^{*}: X_{1}^{*} \rightarrow l_{\infty}\left(2^{n}\right)$ are absolutely summing operators.

It is shown in [8] that for any Banach pair $\left\{X_{0}, X_{1}\right\}$ which is dual to a pair with the approximation property, the space $G_{5}^{\varphi}\left(X_{0}, X_{1}\right)$ coincides with $\varphi_{u}\left(X_{0}, X_{1}\right)$. The pair $\left\{L_{\infty}(M), L_{\infty}^{k}(M)\right\}$ satisfies the conditions above. Indeed, we have already seen that $\left\{L_{\infty}(M), L_{\infty}^{k}(M)\right\}$ is dual to the regular pair $\left\{L_{1}(M), \stackrel{\circ}{W}_{1}^{-k}(M)\right\}$. In [3] it was also shown that there exists a common basis in $L_{1}(M)$ and $\stackrel{\circ}{W}_{1}^{-k}(M)$, which guarantees that this pair has the approximation property.

Therefore we see that

$$
G_{5}^{\varphi}\left(L_{\infty}(M), L_{\infty}^{k}(M)\right)=\varphi_{u}\left(L_{\infty}(M), L_{\infty}^{k}(M)\right)=\left\langle L_{\infty}(M), L_{\infty}^{k}(M)\right\rangle^{\theta, 2}
$$

for $\varphi(s, t)=s^{1-\theta} t^{\theta}$.

Proposition 5. If $\varphi(s, t)=s^{1-\theta} t^{\theta}$, then the space $\varphi_{u}\left(L_{\infty}(M), L_{\infty}^{k}(M)\right)$ coincides with the orbit of $a_{\theta}=\left\{2^{n \theta}\right\}_{n=-\infty}^{\infty}$ with respect to the bounded linear operators mapping $\left\{l_{2}, l_{2}\left(2^{-n}\right)\right\}$ into $\left\{L_{\infty}(M), L_{\infty}^{k}(M)\right\}$, that is, with the space

$$
\operatorname{Orb}\left(a_{\theta}, L\left(\left\{l_{2}, l_{2}\left(2^{-n}\right)\right\} \rightarrow\left\{L_{\infty}(M), L_{\infty}^{k}(M)\right\}\right)\right) .
$$

Proof. If $x \in \varphi_{u}\left(L_{\infty}(M), L_{\infty}^{k}(M)\right)=G_{5}^{\varphi}\left(L_{\infty}(M), L_{\infty}^{k}(M)\right)$, then $x=$ $T\left(a_{\theta}\right)$ where $T:\left\{l_{1}, l_{1}\left(2^{-n}\right)\right\} \rightarrow\left\{L_{\infty}(M), L_{\infty}^{k}(M)\right\}$ is such that $T^{*}$ is absolutely summing from $\left(L_{\infty}(M)\right)^{*}$ into $l_{\infty}$ and from $\left(L_{\infty}^{k}(M)\right)^{*}$ into $l_{\infty}\left(2^{n}\right)$. These operators $T^{*}$ can be factorized through Hilbert spaces and consequently, as already mentioned, the operator $T$ can be factorized through a Hilbert pair $\left\{H_{0}, H_{1}\right\}$, i.e. $T=S_{2} S_{1}$ where $S_{1}:\left\{l_{1}, l_{1}\left(2^{-n}\right)\right\} \rightarrow\left\{H_{0}, H_{1}\right\}$ and $S_{2}:\left\{H_{0}, H_{1}\right\} \rightarrow\left\{L_{\infty}(M), L_{\infty}^{k}(M)\right\}$.

Thus $x=S_{2}\left(S_{1}\left(a_{\theta}\right)\right)$ where $S_{1}\left(a_{\theta}\right) \in\left(H_{0}, H_{1}\right)_{\theta, \infty}$. By $K$-monotonicity of Hilbert pairs (see, for example, [10]) we have $S_{1}\left(a_{\theta}\right)=U\left(a_{\theta}\right)$ where $U:\left\{l_{2}, l_{2}\left(2^{-n}\right)\right\} \rightarrow\left\{H_{0}, H_{1}\right\}$. 
Hence $x=S_{2} U\left(a_{\theta}\right)$ where $S_{2} U:\left\{l_{2}, l_{2}\left(2^{-n}\right)\right\} \rightarrow\left\{L_{\infty}(M), L_{\infty}^{k}(M)\right\}$.

Conversely, if $x=W\left(a_{\theta}\right)$ where $W:\left\{l_{2}, l_{2}\left(2^{-n}\right)\right\} \rightarrow\left\{L_{\infty}(M), L_{\infty}^{k}(M)\right\}$, then $x=\widetilde{W}\left(a_{\theta}\right)$ where

$$
\widetilde{W}:\left\{l_{1}, l_{1}\left(2^{-n}\right)\right\} \subset\left\{l_{2}, l_{2}\left(2^{-n}\right)\right\} \rightarrow\left\{L_{\infty}(M), L_{\infty}^{k}(M)\right\} .
$$

By Grothendieck's theorem, $W^{*}$ is absolutely summing as it maps the dual to an $L_{\infty}$-space into a Hilbert space. Hence, $\widetilde{W}^{*}$ is also absolutely summing.

Thus $x \in G_{5}^{\varphi}\left(L_{\infty}(M), L_{\infty}^{k}(M)\right)$. The proposition is proved.

Recall that the definition of $\varphi_{m}\left(X_{0}, X_{1}\right)$ for $\varphi(s, t)=s^{1-\theta} t^{\theta}$ is similar to the definition of $G_{5}^{\varphi}\left(X_{0}, X_{1}\right)$ where the pair $\left\{l_{1}, l_{1}\left(2^{-n}\right)\right\}$ has to be replaced by $\left\{l_{2}, l_{2}\left(2^{-n}\right)\right\}$. Namely $x \in \varphi_{m}\left(X_{0}, X_{1}\right)$ if $x=T\left(a_{\theta}\right)$ where $a_{\theta}=$ $\left\{2^{n \theta}\right\}_{n=-\infty}^{\infty}$ and $T:\left\{l_{2}, l_{2}\left(2^{-n}\right)\right\} \rightarrow\left\{X_{0}, X_{1}\right\}$ is such that $T^{*}: X_{0}^{*} \rightarrow l_{2}$ and $T^{*}: X_{1}^{*} \rightarrow l_{2}\left(2^{n}\right)$ are absolutely summing.

Since the dual operator to any linear bounded operator, mapping a Hilbert space into an $L_{\infty}$-space, is absolutely summing, we conclude that

$$
\operatorname{Orb}\left(a_{\theta}, L\left(\left\{l_{2}, l_{2}\left(2^{-n}\right)\right\} \rightarrow\left\{L_{\infty}(M), L_{\infty}^{k}(M)\right\}\right)\right)=\varphi_{m}\left(L_{\infty}(M), L_{\infty}^{k}(M)\right) .
$$

Thus

$$
\begin{aligned}
\left\langle L_{\infty}(M), L_{\infty}^{k}(M)\right\rangle^{\theta, 2} & =\varphi_{u}\left(L_{\infty}(M), L_{\infty}^{k}(M)\right) \\
& =G_{5}^{\varphi}\left(L_{\infty}(M), L_{\infty}^{k}(M)\right) \\
& =\varphi_{m}\left(L_{\infty}(M), L_{\infty}^{k}(M)\right) \\
& =\operatorname{Orb}\left(a_{\theta}, L\left(\left\{l_{2}, l_{2}\left(2^{-n}\right)\right\} \rightarrow\left\{L_{\infty}(M), L_{\infty}^{k}(M)\right\}\right)\right)
\end{aligned}
$$

for $\varphi(s, t)=s^{1-\theta} t^{\theta}$.

5. Interpolation theorems for spaces of continuous functions. As usual let $C(M)$ denote the space of all uniformly continuous bounded functions on $M$. Correspondingly let $C^{k}(M)$ denote the space of all functions whose $k$ derivatives belong to $C(M)$.

The spaces $C(M)$ and $C^{k}(M)$ are $L_{\infty}$-spaces, therefore again by the Grothendieck theorem we see that an analogue of Proposition 4 is valid, i.e.

as well as

$$
\varphi_{u}\left(C(M), C^{k}(M)\right)=\left\langle C(M), C^{k}(M)\right\rangle^{\theta, 2}
$$

$$
\operatorname{Orb}\left(a_{\theta}, L\left(\left\{l_{2}, l_{2}\left(2^{-n}\right)\right\} \rightarrow\left\{C(M), L_{\infty}^{k}(M)\right\}\right)\right)=\varphi_{m}\left(C(M), L_{\infty}^{k}(M)\right) .
$$

Theorem 2. For any $k \in \mathbb{N}$ and any one-dimensional manifold $M$,

$$
\varphi_{u}\left(C(M), C^{k}(M)\right)=\left\langle L_{\infty}(M), L_{\infty}^{k}(M)\right\rangle^{\theta, 2}
$$

where $\varphi(s, t)=s^{1-\theta} t^{\theta}$. 
Proof. Obviously, $C(M)$ is equal to the closure of $L_{\infty}^{k}(M) \cap L_{\infty}(M)$ in $L_{\infty}(M)$. Therefore any operator $T:\left\{l_{2}, l_{2}\left(2^{-n}\right)\right\} \rightarrow\left\{L_{\infty}(M), L_{\infty}^{k}(M)\right\}$ continuously maps $l_{2}$ into $C(M)$. Hence,

$$
\begin{aligned}
\operatorname{Orb}\left(a_{\theta}, L\left(\left\{l_{2}, l_{2}\left(2^{-n}\right)\right\}\right.\right. & \left.\left.\rightarrow\left\{L_{\infty}(M), L_{\infty}^{k}(M)\right\}\right)\right) \\
& =\operatorname{Orb}\left(a_{\theta}, L\left(\left\{l_{2}, l_{2}\left(2^{-n}\right)\right\} \rightarrow\left\{C(M), L_{\infty}^{k}(M)\right\}\right)\right) .
\end{aligned}
$$

Thus

$$
\varphi_{u}\left(L_{\infty}(M), L_{\infty}^{k}(M)\right)=\varphi_{m}\left(L_{\infty}(M), L_{\infty}^{k}(M)\right)=\varphi_{m}\left(C(M), L_{\infty}^{k}(M)\right) .
$$

By the general inclusion $\varphi_{m}\left(X_{0}, X_{1}\right) \subset \varphi_{u}\left(X_{0}, X_{1}\right)$ (see [5]), we have

$$
\varphi_{m}\left(C(M), L_{\infty}^{k}(M)\right) \subset \varphi_{u}\left(C(M), L_{\infty}^{k}(M)\right),
$$

and consequently $\varphi_{u}\left(L_{\infty}(M), L_{\infty}^{k}(M)\right) \subset \varphi_{u}\left(C(M), L_{\infty}^{k}(M)\right)$.

The space $L_{\infty}^{k}(M)$ is equal to the Gagliardo completion of $C^{k}(M)$ relative to $L_{\infty}(M)+L_{\infty}^{k}(M)$. From the general property of stability of the functor $\varphi_{u}$ with respect to the Gagliardo completion (see [6]) we deduce that

$$
\varphi_{u}\left(C(M), C^{k}(M)\right)=\varphi_{u}\left(C(M), L_{\infty}^{k}(M)\right) .
$$

Obviously, $\varphi_{u}\left(C(M), L_{\infty}^{k}(M)\right) \subset \varphi_{u}\left(L_{\infty}(M), L_{\infty}^{k}(M)\right)$. Thus

$$
\varphi_{u}\left(C(M), C^{k}(M)\right)=\varphi_{u}\left(C(M), L_{\infty}^{k}(M)\right)=\varphi_{u}\left(L_{\infty}(M), L_{\infty}^{k}(M)\right),
$$

and in view of Proposition 4, $\varphi_{u}\left(C(M), C^{k}(M)\right)=\left\langle L_{\infty}(M), L_{\infty}^{k}(M)\right\rangle^{\theta, 2}$. The theorem is proved.

Corollary. For $k=1,2$ and $\varphi(s, t)=s^{1-\theta} t^{\theta}$,

$$
\varphi_{m}\left(C(M), L_{\infty}^{k}(M)\right) \cap C(M)=\varphi_{u}\left(C(M), C^{k}(M)\right) \cap C(M)=\mathrm{bmo}^{k \theta}(M) .
$$

Thus bmo ${ }^{\theta}(M)$ is an interpolation space between $C^{1}(M)$ and $C(M)$ if $0<\theta<1$, and between $C^{2}(M)$ and $C(M)$ if $0<\theta<2$. It looks very plausible that equalities similar to the latter one and Theorem 1 are still valid for other integers $k$. It is necessary to use a lifting and an analogue of Wolff's theorem for spaces $\varphi_{u}\left(L_{\infty}(M), L_{\infty}^{k}(M)\right)$ (see [8]).

The case of manifolds of higher dimensions. As noticed by Strichartz [13], the trace of the space $\operatorname{bmo}^{\theta}\left(\mathbb{R}^{2}\right)$ on $\mathbb{R}$ coincides with the HölderZygmund space $\mathcal{C}^{\theta}(\mathbb{R})$ for $\theta>0$. Therefore the operator $\mathcal{P}: x(t, s) \mapsto$ $x(t, 0)$ maps boundedly $C\left(\mathbb{R}^{2}\right)$ into $C\left(\mathbb{R}^{2}\right)$ and $C^{k}\left(\mathbb{R}^{2}\right)$ into $C^{k}\left(\mathbb{R}^{2}\right)$, but $\mathcal{P}: \operatorname{bmo}^{\theta}\left(\mathbb{R}^{2}\right) \nrightarrow \operatorname{bmo}^{\theta}\left(\mathbb{R}^{2}\right)$. Thus, $\operatorname{bmo}^{\theta}\left(\mathbb{R}^{2}\right)$ is not an interpolation space between $C\left(\mathbb{R}^{2}\right)$ and $C^{k}\left(\mathbb{R}^{2}\right)$ for $0<\theta<k$. Similarly it is possible to show that $\operatorname{bmo}^{\theta}\left(\mathbb{R}^{2}\right)$ is not an interpolation space between $L_{\infty}(M)$ and $L_{\infty}^{k}(M)$.

6. Boundedness of commutators. In this section we consider only spaces of functions defined on the real axis, so we omit $\mathbb{R}$ in notation. 
Denote by $L_{\infty}^{\alpha}$, where $0<\alpha<1$, the space of Bessel potentials of order $\alpha$ generated by bounded functions, namely $L_{\infty}^{\alpha}=J_{\alpha}\left(L_{\infty}\right)$.

It was shown in [11] that the operator $a H-H a$, where $H$ is the Hilbert transform, is bounded from $L_{p}$ into $W_{p}^{\alpha}$ if $a \in L_{\infty}^{\alpha}$. With the help of Theorem 1 it is possible to get a straightforward proof of a somewhat stronger statement.

Theorem 3. If $a \in$ bmo $^{\alpha}$, then the operator $a H-H a$ maps boundedly $L_{p}$ into $W_{p}^{\alpha}$ for any $1<p<\infty$.

Proof. Let $f \in L_{p}$, then $a H(f)-H(a f) \in L_{p}$ for any $a \in L_{\infty}$. Hence each $f \in L_{p}$ generates a linear operator from $L_{\infty}$ into $L_{p}$, and

$$
\|a H(f)-H(a f)\|_{L_{p}} \leq C\|f\|_{L_{p}}\|a\|_{L_{\infty}} .
$$

If $a \in L_{\infty}^{1}$, that is, if we take a Lipschitz function $a$, then by the Calderón theorem [2], $a H(f)-H(a f) \in \dot{W}_{p}^{1}$ where $\dot{W}_{p}^{1}$ denotes the homogeneous Sobolev space, and

$$
\|a H(f)-H(a f)\|_{\dot{W}_{p}^{1}} \leq C\|f\|_{L_{p}}\left\|a^{\prime}\right\|_{L_{\infty}} .
$$

If we apply the functor $\varphi_{u}$ with $\varphi(s, t)=s^{1-\alpha} t^{\alpha}$ to the operator $a \mapsto$ $a H(f)-H(a f)$, then

$$
\|a H(f)-H(a f)\|_{\varphi_{u}\left(L_{p}, \dot{W}_{p}^{1}\right)} \leq C\|f\|_{L_{p}}\|a\|_{\varphi_{u}\left(L_{\infty}, L_{\infty}^{1}\right)},
$$

and

$$
\|a H(f)-H(a f)\|_{\varphi_{u}\left(L_{p}, \dot{W}_{p}^{1}\right) \cap L_{p}} \leq C\|f\|_{L_{p}}\|a\|_{\varphi_{u}\left(L_{\infty}, L_{\infty}^{1}\right) \cap L_{\infty}} .
$$

In the case of $1<p<\infty$ the pair $\left\{L_{p}, \dot{W}_{p}^{1}\right\}$ is rather simple, since it is a retract of a pair of reflexive lattices. Therefore $\varphi_{u}\left(L_{p}, \dot{W}_{p}^{1}\right)$ coincides with the complex method space $\left[L_{p}, \dot{W}_{p}^{1}\right]_{\alpha}$, that is, with $\dot{W}_{p}^{\alpha}$.

Thus in view of $W_{p}^{\alpha}=L_{p} \cap \dot{W}_{p}^{\alpha}$ we conclude that

$$
\|a H(f)-H(a f)\|_{W_{p}^{\alpha}} \leq C\|f\|_{L_{p}}\|a\|_{\mathrm{bmo}^{\alpha}}
$$

by Theorem 1 and Proposition 4 . The theorem is proved.

\section{References}

[1] J. Bergh and J. Löfström, Interpolation Spaces. An Introduction, Springer, Berlin, 1976.

[2] A. P. Calderón, Commutators, singular integrals on Lipschitz curves and applications, in: Proc. Internat. Congress Math. Helsinki, 1978, Vol. 1, 1980, 85-96.

[3] Z. Ciesielski and T. Figiel, Spline bases in classical function spaces on compact manifolds, Studia Math. 76 (1983), 1-58. 
[4] B. Mitiagin and E. M. Semenov, Absence of interpolation of linear operators in spaces of smooth functions, Izv. Akad. Nauk SSSR 41 (1977), 1229-1266 (in Russian); English transl. Math. USSR-Izv. 11 (1977), 1289-1328.

[5] V. I. Ovchinnikov, Interpolation theorems, resulting from the Grothendieck inequality, Funktsional. Anal. i Prilozhen. 10 (1976), no. 4, 45-54 (in Russian); English transl.: Functional Anal. Appl. 10 (1977), 287-294.

[6] —, The method of orbits in interpolation theory, Math. Reports 1 (1984), 349-516.

[7] - Interpolation properties of fractional BMO space, in: All-Union School on the Theory of Operators in Functional Spaces, Kuibyshev, 1988, 142 (in Russian).

[8] -, On reiteration theorems, in: Voronezh Winter Mathematical Schools, P. Kuchment and V. Lin (eds.), Amer. Math. Soc., Providence, R.I., 1998, 185-198.

[9] G. Pisier, Factorization of Linear Operators and Geometry of Banach Spaces, CBMS Regional Conf. Ser. in Math. 60, Amer. Math. Soc., Providence, R.I., 1986.

[10] A. A. Sedaev, Description of interpolation spaces of the pair $\left(L_{\alpha_{0}}^{p}, L_{\alpha_{1}}^{p}\right)$ and some related questions, Dokl. Akad. Nauk SSSR 209 (1973), 798-800 (in Russian); English transl. in Soviet Math. Dokl. 14 (1973).

[11] C. Segovia and R. L. Wheeden, Fractional differentiation of the commutator of the Hilbert transform, J. Funct. Anal. 8 (1971), 341-359.

[12] R. S. Strichartz, Bounded mean oscillation and Sobolev spaces, Indiana Univ. Math. J. 29 (1980), 539-558.

[13] —, Traces of BMO-Sobolev spaces, Proc. Amer. Math. Soc. 83 (1981), 509-513.

[14] H. Triebel, The Theory of Function Spaces, Birkhäuser, Basel, 1983.

Mathematical Department

Voronezh State University

Universitetskaia pl., 1

394693, Voronezh, Russia

E-mailvio@func.vsu.ru 